\title{
Spectral dependences of the principal angle of incidence and quasi polarizing angle of metals
}

\author{
A. W. Abdallah*, N. A. Mahmoud, N. N. Nagib \\ National Institute of Standards (NIS), Tersa St. Haram, P.O. Box: 136 Giza 12211, Egypt \\ ${ }^{(*)}$ E-mail: alshaimaa.waheed@nis.sci.eg \\ Received: 28/10/2021 Accepted: 21/12/2021 \\ DOI: $10.7149 /$ OPA.54.4.51073
}

\begin{abstract}
:
Principal angle of incidence and quasi-polarizing angle methods are performed to determine the spectral dependences of the principal angle of incidence and quasi-polarizing angle of copper polycrystalline metal at range of wavelengths from $500 \mathrm{~nm}-650 \mathrm{~nm}$. Using the principal angle of incidence method, the spectral dependences of the optical constants of copper sample are determined. In case of the quasi-polarizing angle method, the intensity of the reflection of p-polarized component is measured at different angles of incidence for each wavelength. The angle of incidence at which the intensity has minimum value is referred to the quasi polarizing angle for this wavelength. The results show that the quasi polarizing angle is found to be close to the value of the principal angle of incidence for the copper sample at each wavelength.
\end{abstract}

Key words: Principal angle of incidence, Copper polycrystalline material, Optical constants, Quasi polarizing angle.

\section{REFERENCES}

[1] E. D. Palik, "Handbook of optical constants of solids", (1998)

[2] T. Babeva, S. Kitova and I. Konstantinov, "Photometric method for determining the optical constants and the thicknesses of thin absorbing films: criteria for precise and unambiguous determination of $\mathrm{n}, \mathrm{k}$, and d in a wide spectral range", Appl. Opt. 40 2682-6 (2001).

[] M. Shehata, A. W. Abdallah, S. S. Ibrahim, M. H. Osman, N. N. Nagib, “Determination of a grown oxide layer thickness and optical constants of Zn and Cd metals", Optik, 232, 166552 (2021).

[4] S. M. Al-Shomar, M. A. Y. Barakat, A. W. Abdallah, "Ellipsometric and ultrasonic studies of nano titanium dioxide specimens doped with Erbium", Materials Research Express, 7(10) (2020).

[5] R. M. A. Azzam and E. Ugbo, "Contours of constant pseudo Brewster angle in the complex plane and an analytical method for the determination of optical constants," Appl. Opt. 28, 5222-5228 (1989).

[6] A. W. Abdallah, R. Tutsch and N. N. Nagib, "Novel wide-angle ellipsometric arrangement for thin film thickness Measurement", J. Phys. Commun. 2055007 (2018).

[7] M. Born and E. Wolf, "Principles of Optics", 7th edn (Cambridge: Cambridge University Press), (1999)

[] H. G. Tompkins and E. A. Irene, "Handbook of Ellipsometry", (2005).

[9] A. C. Hall, "Experimental determination of the optical constants of metals" J. Opt. Soc. Am. 55 9115(1965).

[10] N. N. Nagib, M. S. Bahrawi, H. Osman, N. A. Mahmoud, M. H. Osman, A. W. Abdallah, "A precise method for determining the principal angle of incidence and the optical constants of metals", Meas. Sci. Technol. 27015009 (2016).

[11] S. Babar and J. H. Weaver, "Optical constants of Cu, Ag and Au”, revisited Appl. Opt. 54 477-81 (2015).

[12] P. B. Johnson and R. W. Christy, “Optical constants of the noble metals”, Phys. Rev. 6 4370-9 (1972).

[13] A. W. Abdallah, "Applications of polarized light in optical metrology" MSc Thesis Physics Department, Faculty of Science, Cairo University (2014). 
[14] R. M. A. Azzam and A. Alsamman, "Plurality of principal angles for a given pseudo Brewster angle when polarized light is reflected at a dielectric conductor interface," J. Opt. Soc. Am. A 25, 2858-2864 (2008).

[15] N. N. Nagib, M. S. Bahrawi, L. Z. Ismail, M. H. Othman, A. W. Abdallah, "Polarization metrology: Alignment of polarizing prisms in optical polarization systems", Optics \& Laser Technology 54 42-44 (2013).

[16] M. Akimoto, Y. Gekka, "Brewster and pseudo Brewster angle technique for determination of optical constants", Jpn. J. Appl. Phys. 31 pp. 120-122 (1992).

[17] T. E. Darcie, M. S. Whalen, "Determination of optical constants using pseudo Brewster angle and normal incidence reflectance measurements", Applied Optics (1984).

[18] M. A. Ordal, R. J. Bell, R. W. Alexander, L. L. Long and M. R. Querry, "Optical properties of fourteen metals in the infrared and far infrared: $\mathrm{Al}, \mathrm{Co}, \mathrm{Cu}, \mathrm{Au}, \mathrm{Fe}, \mathrm{Pb}, \mathrm{Mo}, \mathrm{Ni}, \mathrm{Pd}, \mathrm{Pt}, \mathrm{Ag}, \mathrm{Ti}, \mathrm{V}$ and W", Appl. Opt. 24 4493-9 (1985).

\section{Introduction}

Optical constants of metals can be determined by different methods such as ellipsometry, principal angle of incidence method, photometric method and pseudo-Brewster angle method [1-6]. The traditional principal angle of incidence method for determination of the optical constants of a metal is described in [7, 8]. Plane polarized light at azimuth $45^{\circ}$ is incident by an angle of incidence $\phi$ on a metal surface and then reflected to the other arm which consists of quarter wave plate set at $0^{\circ}$, analyzer rotate to extinction and detector for intensity measurements. At fixed angle of incidence $\phi$, the phase shift is considered as $\Delta=\delta_{p}-\delta_{s}$ and the amplitude changetan $\psi=\left|r_{p} / r_{s}\right|$. The angle of incidence is changed and analyzer angle is adjusted to reach minimum intensity. At a definite angle of incidence, the light can be extinguished by rotating the analyzer setting to a certain angle, this extinction occurs when the polarization ellipse reflected from the metal surface reaches a standard form and represents a phase shift equal to $90^{\circ}$. The angle of incidence corresponding to extinction is called the principal angle of incidence $\phi_{i}$, and the principal azimuthal angle $\Psi$ is represented by the analyzer angle [9]. This method has many defects and sources of errors as described in [10], so a modified principal angle of incidence method of two steps procedure is used for measuring the optical constants of copper thin film at single wavelength, this method overcomes most of the sources of error that were found in the traditional method [11]. The first step is by removing the quarter wave plate from the optical setup (polarizer-sample-analyzer). The angle of incidence is changed with respect to the sample and analyzer angle is rotated for maximum intensity. The angle of incidence corresponding to analyzer reading $90^{\circ}$ is known as the principal angle of incidence $\phi_{i}$. The second step is to insert the quarter wave plate after the sample with the setting of angle to the principal angle of incidence $\phi_{i}$, then the principal azimuthal angle $\Psi$ is determined when rotating the analyzer angle at extinction. The optical constants (n, k) can be calculated from the following equations [12]:

$$
\begin{gathered}
n=-\sin \phi_{i} \tan \phi_{i} \cos 2 \Psi \\
k=-\tan 2 \Psi
\end{gathered}
$$

The success of this modified method is presented in [11] to measure the optical constants of copper thin film at single wavelength.

In this work, the spectral dependence of the principal angles of incidence and quasi polarizing angles of copper polycrystalline metal are determined in the wavelength range from $500 \mathrm{~nm}$ and $650 \mathrm{~nm}$ using the quasi-polarizing angle and the principal angle of incidence methods. The results are compared to previous publications for measuring the optical constants of copper as in $[11,13]$. The quasi-polarizing angle method, is defined as the angle of incidence at which the intensity is reached to its minimum value and is supposed to be close to the value of the principal angle of incidence [14]. The quasi-polarizing angles are measured at wavelengths range from $500 \mathrm{~nm}-650 \mathrm{~nm}$. Our goal in this research is to further assess the utility of the modified principal angle of incidence method at range of wavelengths from $500 \mathrm{~nm}$ to $650 \mathrm{~nm}$ and to identify the difference between the values of the principal angle of incidence and quasi polarizing angle of metals. 


\section{Experimental work}

Fig. 1(a) shows the optical arrangement of the principal angle of incidence method, the first step of this method: a monochromatic beam $(\lambda)$, which is obtained using Double monochromator source model MSA130 Solar Laser Systems. The beam passes through a polarizar adjusted at $45^{\circ}$, and then incident with an angle $\Phi$ on the copper polycrystalline metal surface (sheets of purity $99.999 \%$, thickness $\sim 0.05 \mathrm{~mm}$ and surface area $30 \mathrm{~mm} \times 30 \mathrm{~mm}$ ) that is placed on a rotating stage. The reflected beam passes through an analyzer and then the output intensity is detected by using photomultiplier detector. In the beginning of the experiment, calibration of both polarizer $P$ and analyzer $A$ is performed as in [15].

The first step of the modified method is to remove the quarter wave plate from the setup and change the angle of incidence by rotating the sample stage from $60^{\circ}$ to $74^{\circ}$ in steps of $2^{\circ}$. For each angle, the analyzer setting is adjusted for maximum value of the output intensity. The angle at which the analyzer setting is $90^{\circ}$ (the polarization ellipse reflected from the metal surface reaches a standard form) is called the principal angle of incidence $\phi_{i}$.

The second step of the method is illustrated in fig. 1 (b). The quarter wave plate is inserted in the optical setup after the sample. The angle of incidence of the sample is adjusted to the principal angle of incidence $\phi_{i}$. The reflected beam is passes through a quarter wave plate with its fast axis at $0^{\circ}$ and then incident on an analyzer and a detector. The analyzer angle is rotated until the light intensity is extinguished. The angle of analyzer setting at extinction is known as the principal azimuthal angle $\Psi$.

The two steps of the modified method are repeated for a range of wavelengths from $500 \mathrm{~nm}$ to $650 \mathrm{~nm}$ in steps of $50 \mathrm{~nm}$. For each wavelength, principal angle of incidence and principal azimuthal angle are determined. From these angles, the optical constants of polycrystalline copper metal are calculated.

Fig. 2 represents the optical arrangement of the quasi polarizing angle measurement, where a monochromatic beam of wavelength $\lambda$ is incident on a polarizer with azimuth angle $45^{\circ}$ and then incident on a metal surface with an angle of incidence $\phi$, the reflected beam is passes through analyzer with azimuth angle $0^{\circ}$ and incident on the photomultiplier detector. The angle of incidence is changed from $65^{\circ}-77^{\circ}$ in steps of $2^{\circ}$ and the output intensity is recorded using a digital multimeter for each angle. The intensity of the reflected p-polarized component at a range of angles of incidence is measured [16]. The angle of incidence at which the output intensity reaches its minimum value refers to the quasi polarizing angle. This angle is measured at range of wavelengths from $500 \mathrm{~nm}$ to $650 \mathrm{~nm}$ in steps of $50 \mathrm{~nm}$.

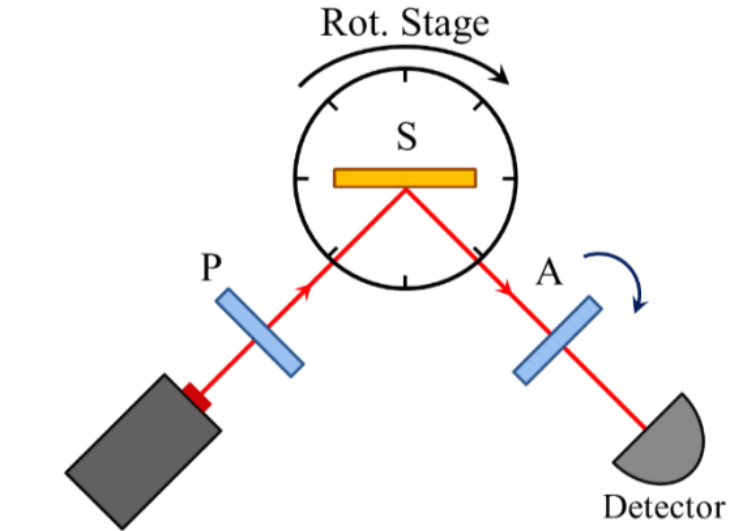

Monochromator

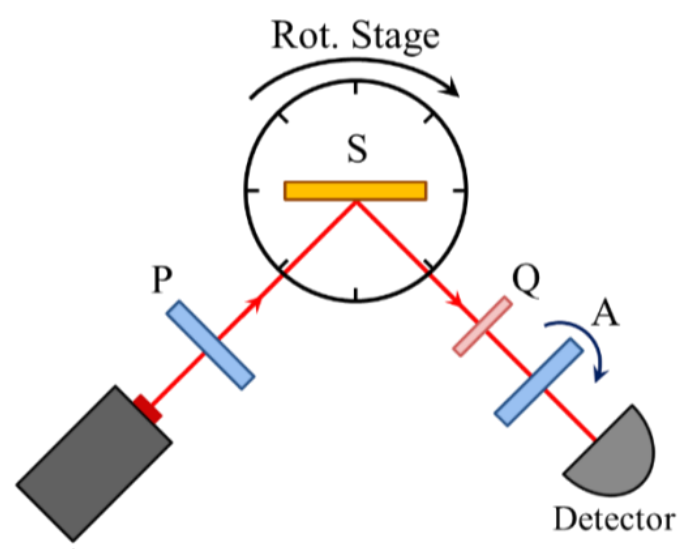

Monochromator

Fig. 1. Optical arrangement of the modified principal angle of incidence. It consists of Double Monochromator, P, A: polarizi ng elements, S: sample, Q: quarter wave plate and Photomultiplier Detector (a) first step (b) second step. 


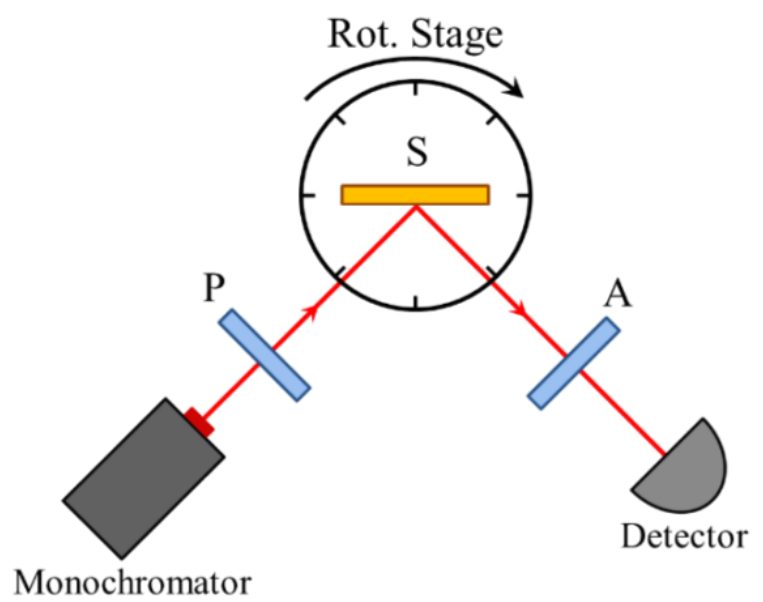

Fig. 2. Optical arrangement of the quasi polarizing method.

\section{Results and discussion}

As illustrated in Fig. 1 (a) the angles of incidence varied between $60^{\circ}-74^{\circ}$ in steps of $2^{\circ}$. For each angle, the analyzer setting is rotated until we get maximum intensity. Fig. 3 shows the relation between the analyzer reading at maximum and different angles of incidence for copper polycrystalline sample at wavelength (a) $500 \mathrm{~nm}$, (b) $550 \mathrm{~nm}$, (c) $600 \mathrm{~nm}$, (d) $650 \mathrm{~nm}$. The principal angle of incidence is the angle of incidence related to the analyzer setting 90 ․ From Fig. 3 the principal angles of incidence are determined in the spectral range $500 \mathrm{~nm}$ to $650 \mathrm{~nm}$ for copper polycrystalline metal.

In Fig 1. (b) For each wavelength, the sample stage is adjusted to the principal angle of incidence and then the analyzer angle is rotated for extinction, this angle refers to the principal azimuthal angle. Table.1 illustrates the spectral dependence of the principal angle of incidence, principal azimuthal angle and optical constants $\mathrm{n}, \mathrm{k}$ in the wavelength range $500 \mathrm{~nm}-650 \mathrm{~nm}$.

Fig. 4 represents the spectral dependence of the principal angle of incidence and principal azimuthal angle of copper polycrystalline metal in the wavelength range $500 \mathrm{~nm}-650 \mathrm{~nm}$.

Table. 2 shows values of the optical constants of copper in this work and in the literature. The results are in a good agreement with the literature. The observed derivations between our results and other publications are most likely due to the different nature of samples (thin film or polycrystalline) used, the preparation method [18].

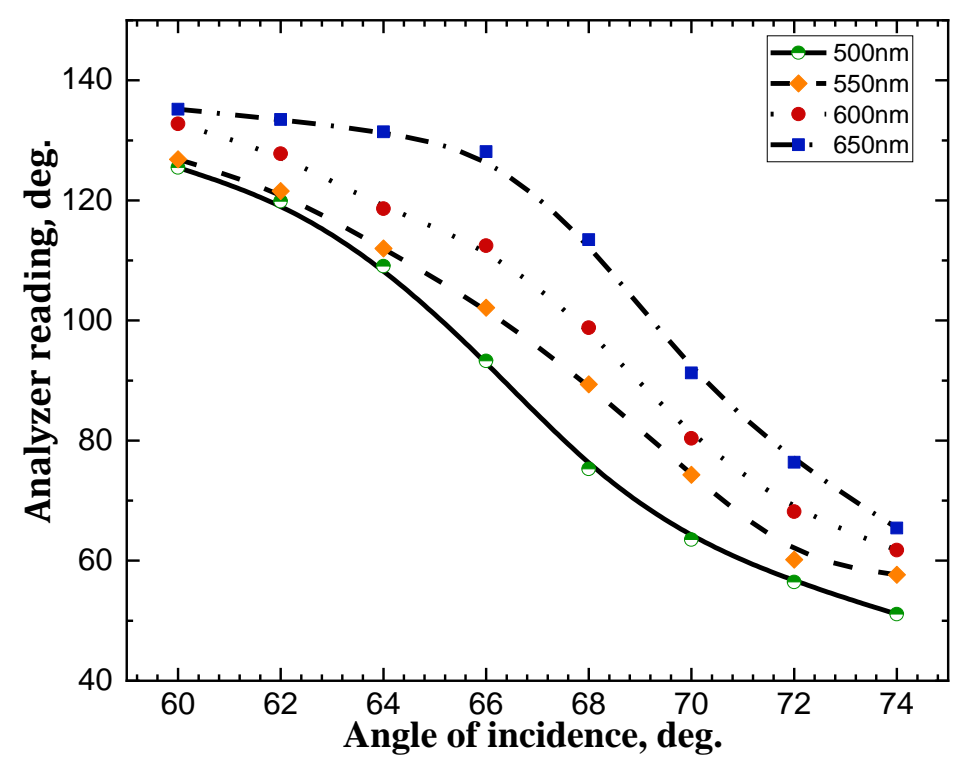

Fig. 3 The analyzer readings at different angles of incidence for copper polycrystalline sample in the case of the wavelengths: $500 \mathrm{~nm}$, $550 \mathrm{~nm}, 600 \mathrm{~nm}$ and $650 \mathrm{~nm}$. 
TABLE.1 The spectral dependences of the principal angle of incidence, principal azimuthal angle and optical constants $\mathrm{n}$, $\mathrm{k}$ in the wavelength range $500 \mathrm{~nm}-650 \mathrm{~nm}$.

\begin{tabular}{|c|c||c|c|c|}
\hline$\lambda, \mathbf{n m}$ & $\phi_{i}$, deg. & $\Psi$, deg. & $\boldsymbol{n}$ & $\boldsymbol{k}$ \\
\hline 500 & 66.65 & 143.33 & 0.622 & 3.342 \\
\hline 550 & 68.45 & 142.33 & 0.6101 & 3.823 \\
\hline 600 & 69.3 & 141.58 & 0.584 & 4.277 \\
\hline 650 & 70.3 & 140 & 0.547 & 4.705 \\
\hline
\end{tabular}

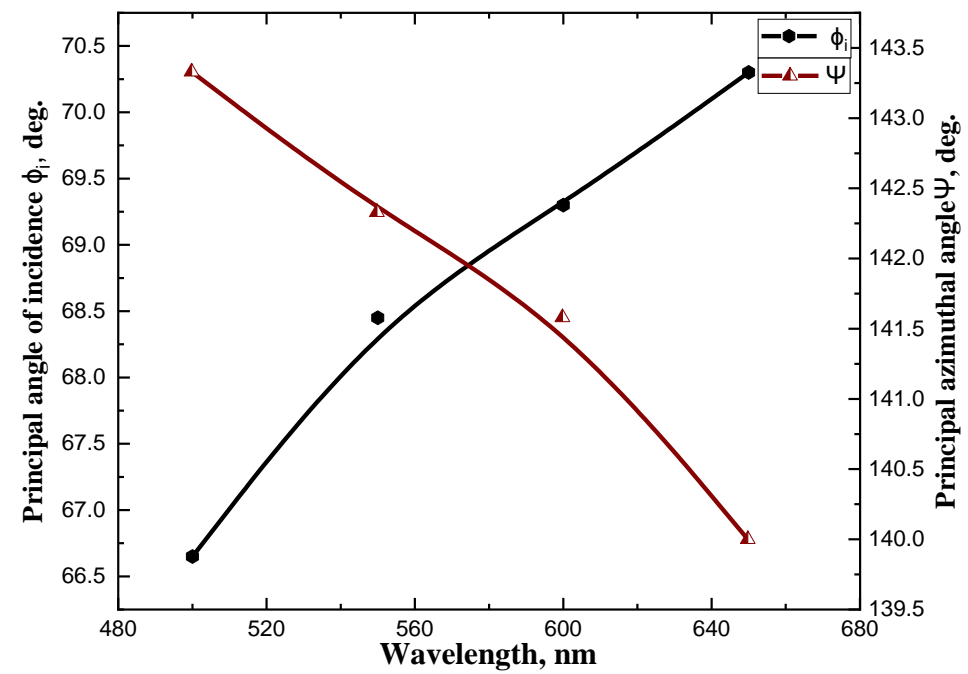

Fig. 4 The relation between the principal angle of incidence and the principal azimuthal angle with wavelength, $\mathrm{nm}$.

TABLE. 2 The optical constants of copper at $632.8 \mathrm{~nm}$ from this work and literature.

\begin{tabular}{||c|c|c|}
\hline $\mathbf{n}$ & $\mathbf{k}$ & Literature \\
\hline & & \\
0.2700 & 3.4081 & {$[12]$} \\
0.3070 & 3.4345 & {$[19]$} \\
0.5840 & 3.6466 & {$[18]$} \\
0.6625 & 4.8459 & {$[10]$} \\
0.5527 & 4.6831 & From this work \\
\hline
\end{tabular}

The traditional principal angle of incidence is more complicated and requires a lot of trials to determine both the principal angle of incidence and principal azimuthal angle at the same time [11]. The modified principal angle of incidence method is simple and the results are of high accuracy than the traditional one.

Fig. 5 shows the intensity of the reflected p-polarized component measured at angles of incidence range from $65^{\circ}-77^{\circ}$ for wavelengths (a) $500 \mathrm{~nm}$, (b) $550 \mathrm{~nm}$, (c) $600 \mathrm{~nm}$ and (d) $650 \mathrm{~nm}$. Polynomial fit analysis is performed for each curve as represented in Fig. 5. For each wavelength, the angle of incidence related to the minimum value of the output intensity is refer to the quasi polarizing angle. 

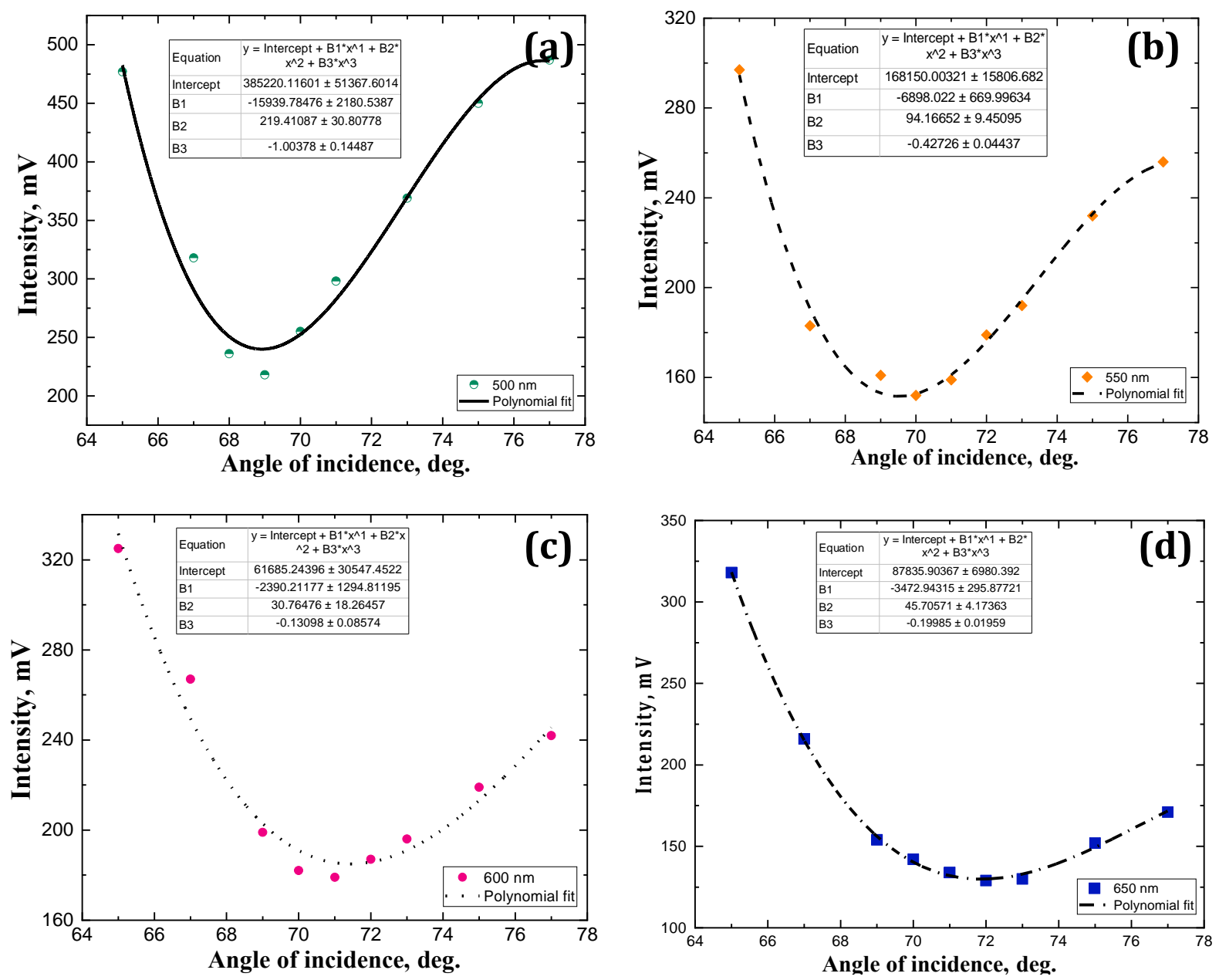

Fig. 5 Shows the relation between the intensity of reflected p-polarized component and angles of incidence at different wavelengths (a) $500 \mathrm{~nm}$, (b) $550 \mathrm{~nm}$, (c) $600 \mathrm{~nm}$ and (d) $650 \mathrm{~nm}$.

At wavelength $500 \mathrm{~nm}$, the third polynomial fit equation is as followed:

$$
f\left(\varphi_{q}\right)=385220.1162-15939.785 \varphi_{q}+219.41 \varphi_{q}^{2}-1.0038 \varphi_{q}^{3}
$$

Taking the first derivative of (3) and equal it $6 \mathrm{oz}$ ero:

$$
0=15939.785-438.82 \varphi_{q}+3.0114 \varphi_{q}^{2}
$$

Then by solving this equation, we get the roots:

$$
\varphi_{q 1}=76.7844^{\circ}, \quad \varphi_{q 2}=68.9352^{\circ}
$$

As illustrated in Fig. 4 the root $\varphi_{q 2}$ value is more realistic to express the 6oz ero value represented in the curve of fitting and is closer to the principal angle of incidence value at this wavelength, so the quasi polarizing angle at wavelength $500 \mathrm{~nm}$ is $68.9352^{\circ}$.

The same steps are occurred for all wavelengths, so the third polynomial equation and the roots after derivative the equation and equating it 6oz ero, is as illustrated below:

At wavelength $550 \mathrm{~nm}$ :

$$
\begin{gathered}
f\left(\varphi_{q}\right)=168150.0032-6898.0223 \varphi_{q}+94.1665 \varphi_{q}^{2}-0.4272 \varphi_{q}^{3} \\
\varphi_{q 1}=77.4114^{\circ}, \quad \varphi_{q 2}=69.5193^{\circ}
\end{gathered}
$$

The quasi polarizing angle at wavelength $550 \mathrm{~nm}$ is $69.5193^{\circ}$ 
At wavelength $600 \mathrm{~nm}$ :

$$
\begin{gathered}
f\left(\varphi_{q}\right)=61685.2439-2390.2117 \varphi_{q}+30.76476 \varphi_{q}^{2}-0.13098 \varphi_{q}^{3} \\
\varphi_{q 1}=85.1512^{\circ}, \quad \varphi_{q 2}=71.4363^{\circ}
\end{gathered}
$$

The quasi polarizing angle at wavelength $600 \mathrm{~nm}$ is $71.4363^{\circ}$

At wavelength $650 \mathrm{~nm}$ :

$$
\begin{gathered}
f\left(\varphi_{q}\right)=87835.9037-3472.9432 \varphi_{q}+45.7057 \varphi_{q}^{2}-0.19985 \varphi_{q}^{3} \\
\varphi_{q 1}=80.5852^{\circ}, \quad \varphi_{q 2}=71.8814^{\circ}
\end{gathered}
$$

The quasi polarizing angle at wavelength $650 \mathrm{~nm}$ is $71.8814^{\circ}$

The quasi polarizing angle can be measured within $0.05^{\circ}$ depending on the experimental parameters of the relative reflectance versus angles of incidence and it doesn't depend on absolute intensity measurements, so this technique is of high accuracy than any other reflection based techniques [17].

\section{Conclusions}

The modified principal angle of incidence method is used to measure the spectral dependence of the principal angles of incidence and principal azimuthal angles of copper polycrystalline sample at wavelengths range from $500 \mathrm{~nm}$ to $650 \mathrm{~nm}$. The optical constants of copper sample are extracted from the measurements of the principal angles of incidence and principal azimuth angles for each wavelength. The quasi polarizing angle method is used for measuring the quasi polarizing angle of the copper sample at each wavelength and compare it with the principal angle of incidence. The quasi polarizing angles are measured from the relation between the intensity of reflection of $\mathrm{p}$ - component with the angles of incidence and the results show that its values are found to be close to the measured principal angles of incidence at each wavelength. 